\title{
Yod
}

Revue des études hébraïques et juives

15 | 2010

Philosophie et pensée juives : histoire et actualité

\section{Sujet, être, être-là : Heidegger, ou l'être humain entre pensée juive et philosophie grecque}

Subject, Being, Being-there: Heidegger or the Human Being between Jewish Thinking and Greek Philosophy

Josef Schovanec

\section{OpenEdition}

Journals

Édition électronique

URL : https://journals.openedition.org/yod/677

DOI : 10.4000/yod. 677

ISSN : 2261-0200

Éditeur

INALCO

Édition imprimée

Date de publication : 1 janvier 2010

Pagination : 235-259

ISBN : 978-2-85831-183-5

ISSN : 0338-9316

Référence électronique

Josef Schovanec, « SUJET, ÊTRE, ÊTRE-LÀ : HEIDEgger, OU L'ÊTRE HUMAIN ENTRE PENSÉE JUIVE ET

PHILOSOPHIE gRecque », Yod [En ligne], 15 | 2010, mis en ligne le 30 octobre 2011, consulté le 08 juillet 2021. URL : http://journals.openedition.org/yod/677 ; DOI : https://doi.org/10.4000/yod.677

Ce document a été généré automatiquement le 8 juillet 2021.

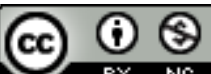

Yod est mis à disposition selon les termes de la Licence Creative Commons Attribution - Pas d'Utilisation Commerciale 4.0 International. 


\title{
Sujet, être, être-là : Heidegger, ou l'être humain entre pensée juive et philosophie grecque
}

\author{
Subject, Being, Being-there: Heidegger or the Human Being between Jewish \\ Thinking and Greek Philosophy
}

Josef Schovanec

\section{Introduction : les deux conceptions idéal-typiques de l'être humain}

1 Be-tsalmo, à l'image de Dieu'. La définition de l'homme est sans doute l'une des expressions les plus connues de la Bible, l'une de celles qui figurent dans les catéchismes qui ont marqué la jeunesse de tant de penseurs occidentaux, tout en faisant preuve de cette étrange concision dont Auerbach ${ }^{2}$ soulignait la nature spécifiquement biblique, distincte du style littéraire grec puis européen, à la fois d'une profondeur insondable et d'une tonalité angoissante.

2 La présente définition se caractérise également par son statut d'exceptionnalité. Exceptionnalité de par la rareté des définitions de l'homme dans la Bible, qui n'est pas un ouvrage systématique au sens actuel du terme, mais également du fait que les religions, en particulier anciennes, proches ou issues de l'univers biblique, régissent avec un soin particulier la notion d'image, l'encadrant de nombre d'interdits : l'unicité du statut de l'homme, image de Dieu, n'en ressort que plus fortement.

Ainsi, la conception biblique de l'être humain peut dès à présent être caractérisée par deux points : son statut exceptionnel vis-à-vis du reste de la création (l'étant, dans le lexique qui sera celui de la présente étude), son lien particulier, difficile à caractériser autrement que de manière elliptique ou poétique, avec l'être divin créateur.

Distincte, voire opposée à cette première tradition, s'est peu à peu constituée une deuxième conception de l'être humain, celle que nous désignerons par la suite sous le 
terme de «sujet ». Elle nous est probablement plus familière, de par le rôle central qu'elle joue dans les sciences de la société, humaines ou de l'esprit, ainsi que dans la philosophie ou des disciplines mixtes telles que la médecine, un rôle qui s'est encore accentué depuis une cinquantaine d'années par l'introduction de différents modèles du « sujet rationnel », issus du paradigme économique, dans diverses sciences de l'esprit telles que la sociologie ou la science politique ${ }^{3}$.

5 S'il ne nous appartient pas de la définir ici avec précision, tâche hautement problématique au vu de ses nombreuses formes, variantes théoriques, disciplinaires ou simplement lexicales, nous pouvons néanmoins préciser sa genèse. Marcuse l'inscrit dans les premiers temps du "monde de la modernité », marqué par le principe de rationalité, qui défait les facultés dites inférieures, s'érige en principe de réalité et de ce fait " étend son pouvoir sur l'Éros ", cet Éros qui était avant Platon la source de toute culture, que Marcuse qualifie d'«archaïque-mystique $»^{4}$. Certains autres philosophes préfèrent fixer la rupture grecque à l'époque de Socrate, d'autres enfin sous Aristote. L'helléniste Marcel Detienne décrit le "processus de laïcisation » qui allait aboutir à une conception de l'homme proche de notre «sujet » comme le fait d'un milieu social ayant résisté à l'emprise de la parole magico-religieuse des maîtres de vérité de la Grèce archaïque, à savoir les poètes, les voyants, les rois : celui des guerriers ${ }^{5}$, un milieu appelé à une expansion numérique rapide suite à la réforme hoplitique qui a transformé les guerriers en citoyens tout en étendant à l'ensemble de la cité un mode de parole spécifique : la véracité du discours y était subordonnée non à la qualité de sa source ou à la complexité du rituel l'accompagnant, mais à la « ratification du groupe social $\aleph^{6}$ qui entourait l'orateur dûment muni du sceptre et se tenant au centre du cercle de ses pairs.

6 Au-delà des descriptions des spécificités historiques de l'émergence de l'une ou l'autre des conceptions et en faisant abstraction des divergences portant sur des points de détail entre les auteurs, deux traditions ou visions assez profondément distinctes de l'être humain se dessinent: celle du sujet propre à la philosophie majoritaire en Occident et celle, pour reprendre l'expression de Ron Naiweld, de l'«anti-sujet ", rabbinique ou non ${ }^{7}$. Toutes deux semblent fonder ou articuler une conception du monde, voire une manière de penser et d'être différentes. Il convient, pour la suite de notre propos, d'énoncer dès à présent une remarque tenant de la simple prudence méthodologique: une grande réserve semble nécessaire quant à l'usage des dénominations et termes techniques dans les études heideggériennes, en raison de leurs sens secondaires débouchant régulièrement sur de graves malentendus ou polémiques, mais également du fait de leur sollicitation excessive par le passé, ayant par exemple amené tel penseur à se déclarer alternativement philosophe ou nonphilosophe, sociologue ou non-sociologue, penseur du sujet ou adversaire de l'humanisme et ce, selon des critères indépendants du contenu de sa pensée. Dès lors, si la commodité nous impose un choix de vocabulaire, qu'il ne soit pas perçu comme une caractérisation parfaite des conceptions abordées dans les présents développements, mais comme un simple signe vers elles.

7 Ceci étant, les profondes divergences entre les deux conceptions de l'être humain n'impliquent pas, et la question mériterait bien des développements tenant de l'histoire des idées, une stricte séparation géographique et historique entre leurs supports culturels : la prépondérance de l'une sur l'autre n'a jamais été absolue, chaque modèle a influencé et s'est inspiré de l'autre, à maintes reprises un penseur a dû son succès à 
l'usage d'une conception minoritaire voire absente de son environnement intellectuel. Le point de vue, qui sera présenté ici, et qui a été plus longuement débattu ailleurs, portera dès lors sur la présence, au cœur même d'une pensée majeure de la modernité, d'une conception de l'être humain issue de cultures et de contextes bien différents.

8 Le cas de Heidegger est, à cette fin, exceptionnel, tant ce penseur a, si l'on s'en tient à la lettre de son œuvre, insisté sur la spécificité de la philosophie occidentale née en Grèce et n'a guère accordé d'intérêt à d'autres traditions culturelles, pour ne pas même mentionner la part trop abondante polémique autour de ses opinions politiques qui ne le prédisposeraient guère au cosmopolitisme de la pensée. Néanmoins, de récentes, mais approfondies recherches ${ }^{8}$ ont abondamment montré l'ampleur insoupçonnée des relations qu'entretenait Heidegger avec ses collègues et amis étrangers, bien au-delà de ce qui était alors de coutume: ses œuvres portent si clairement la marque de ses sources d'inspiration que d'aucuns l'ont accusé d'avoir mis en place un cryptage stratégique systématisé9. Au-delà de ces considérations biographiques, nous présenterons ici quelques ressemblances entre la conception heideggérienne de l'homme et celle que l'on peut retrouver au sein de l'« anti-sujet » juif ou, peut-être plus spécifiquement encore, au sein de la "religion cosmique» ou "sacralité cosmique ", selon les termes d'Eliade, dont sont issues les pages les plus anciennes de la Bible, qui a été reprise dans les courants mystiques du judaïsme et qui est présente, sous une forme rationalisée et donc hellénisée, dans la pensée juive postérieure à la « réforme rabbinique $»^{10}$.

9 Heidegger a lui-même, dans un ouvrage qui a longtemps servi en France d'introduction à sa pensée et que nous choisissons pour fil conducteur du fait qu'il rassemble et résume bon nombre de ses thèses, à savoir la Lettre sur l'Humanisme, présenté la différence entre la conception occidentale de l'être humain, qualifiée d' « humaniste ", et la sienne, que ses disciples français diront «antihumaniste». La première aurait clairement été présente au temps de la République romaine où l'humanitas était expressément "considérée et poursuivie ». De là, elle a triomphé en Grèce, mais seulement pendant l'hellénisme tardif - bien que, toujours selon Heidegger, cette période soit la seule à laquelle il soit fait référence dès lors qu'il est question de la Grèce $^{11}$. L'autre, la sienne, se présente chez Marx, qui voyait l'essence de l'homme dans la société, et dans le christianisme, qui la voit dans l'histoire du salut, dans l'appel du $\mathrm{Christ}^{12}$. La double invocation de Marx et du Christ, si elle est probablement due à des considérations opportunistes liées à la nature des courants de pensée dominants dans l'immédiat après-guerre, ne doit pas surprendre : certains mécanismes mentaux de ces deux traditions sont effectivement au cœur de la pensée de Heidegger tout en étant issus d'une même source intellectuelle, ainsi que nous y reviendrons. La différence fondamentale entre les deux conceptions tiendrait précisément en l'humanitas, définie comme un apprentissage scolaire, qui «se fonde et fonde une métaphysique ", mot à entendre, pour simplifier grandement, comme philosophie rationnelle qui « oublie la vérité de l'Être » et qui vise à créer un homo humanus, distinct et supérieur à l'homo barbarus environnant.

La Lettre, promise à un grand succès en France, a fortement contribué à de nombreuses discussions autour de ce que l'on dénommait l'anthropologisme ${ }^{13}$. La plupart des penseurs des années soixante et soixante-dix, par exemple Foucault, ont ainsi dénoncé le «sommeil anthropologique » dans lequel les sciences auraient été plongées par la philosophie classique, de Kant ou d'autres. 


\section{L'homme en tant qu'être-là}

11 L'exposé plus systématique qui prendra appui sur les remarques générales introductives venant d'être faites et notamment de la dichotomie entre deux grands modèles, peut se déployer à partir d'une caractéristique majeure de l'« anti-sujet » : le fait que, loin d'être, pour reprendre les termes de Ron Naiweld, une entité finie, autonome au sens kantien du terme, l'être humain soit une émanation, une image au sens le plus large, de l'être divin.

Ce changement de paradigme descriptif de l'être humain peut être considéré comme l'un des principaux points de départ de la pensée de Heidegger (le second, plus tardif puisqu'il date de la période que ses biographes appellent la Kehre, retournement ou conversion, au milieu des années trente, ayant probablement été le recentrage de sa pensée sur l'être). Heidegger introduit un terme nouveau pour désigner l'être humain : Dasein, traduit en français dans les premiers temps par « existence humaine ", ensuite, littéralement, par "être-là ", dans les ouvrages les plus orthodoxes par "être-le-là ", ou tout simplement repris tel quel dans la langue des heideggériens francophones. Le terme semble être un jeu de mots qui admet au moins deux niveaux de lecture : en allemand classique, Dasein désigne ce que l'on pourrait dénommer existence effective, non nécessairement celle de l'être humain ${ }^{14}$. Heidegger, quant à lui, incite à le décomposer en ses composantes linguistiques, $d a$, pouvant être le « là " géographique, mais aussi le « car » logique, et le Sein, l'être par excellence, quête de Heidegger.

L'exégèse du terme Dasein a donné lieu à de nombreuses études qu'il n'est pas directement de notre propos de présenter ici. Notons que le premier Heidegger qualifie lui-même son action par l'expression "analytique fondamentale du Dasein", « analytique » étant à entendre également au sens originel comme décomposition non seulement du signifié, mais aussi du signifiant Dasein, selon l'un de ces jeux de mots sérieux (Worternst) dont Heidegger a fait l'un des ressorts de sa pensée. Sa grand œuvre de jeunesse, Être et Temps, contient déjà plusieurs décompositions possibles du terme (par exemple au § 12).

Heidegger attachait une importance particulière au terme Dasein en lieu et place des mots plus habituels. Les heideggériens ont en mémoire l'épisode de 1929 dit « de Davos ", où le jeune Heidegger s'est opposé à Ernst Cassirer. Les débats se sont focalisés sur la place de la philosophie par rapport aux sciences et, par extension, mais contre toute attente, sur la possibilité de traduire sujet en Dasein et l'inverse. Se réclamant du même Kant, les deux protagonistes se sont accordés sur un grand nombre de points : le sujet tout comme le Dasein sont finis et tendent à s'accomplir dans une universalité (Welt der Formen, monde des formes, ou das Sein), tous deux affirment chercher l'humanisme (certes, en accusant l'autre de faire l'inverse). Invités à harmoniser leur vocabulaire, les deux philosophes n'ont pu se mettre d'accord, notamment de par le refus catégorique de Heidegger de transiger sur l'usage de Dasein, terme résumant à ses yeux toute la spécificité de sa démarche.

15 Au-delà de la simple anecdote, ce débat met en lumière plusieurs particularités de la pensée de Heidegger étrangères à la philosophie majoritaire en Occident. D'une part, le changement de désignation en tant que geste initial, voire initiatique : en passant à l'usage de Dasein, Heidegger rejoint des traditions religieuses ou mystiques où le changement de nom tient lieu de baptême, à l'instar de l'entrée dans les ordres. L'usage 
d'un mot nouveau a souvent un rôle central dans les écrits mystiques, par exemple ceux de Saint Jean de la Croix ou de Sainte-Thérèse d'Avila qui désignent l'être humain par " âme » - un terme qui n'est certes pas apparenté à Dasein, mais qui s'en rapproche en ce qu'il présuppose l'existence d'un " animateur » auquel les âmes sont liées d'une mystérieuse façon qui fait l'objet de toute la quête de leurs auteurs. D'autre part, plus globalement, Heidegger s'éloigne d'un principe majeur de la pensée typique de l'Occident, à savoir l'arbitraire du signifiant selon l'expression de Saussure. Selon celuici, le signifiant, le vocabulaire, n'est qu'un signe vers le signifié et peut être changé en cas de besoin, en fonction du contexte linguistique et culturel ou des envies de l'auteur, par exemple pour éviter des répétitions. Ainsi, la tradition majoritaire en Occident permet de traduire des œuvres de philosophie, la traduction conservant, bien entendu sauf erreur due au traducteur, la même valeur que l'original, au même titre qu'une démonstration de mathématiques pouvant être menée indifféremment en anglais ou en français. Heidegger opte pour un tout autre rapport au langage : le langage adopté par le penseur contient ou non toute la vérité de l'être, et doit donc faire l'objet d'une quête particulièrement poussée, tout comme, dans une traduction religieusement conforme de la Bible, YHVH ne saurait être rendu par Baal ou Tao, quand bien même ces termes recouvriraient des réalités analogues dans la langue ou la culture de destination, précisément du fait que l'usage ou non du Nom sacré contribue fortement à façonner la nature juive ou païenne de la religion de la personne considérée. Cela explique la langue, souvent jugée cryptique, des écrits de Heidegger, ses particularités grammaticales, ses jeux étymologiques pour découvrir le vrai, mais aussi la difficulté de le traduire et les interminables querelles de traducteurs. Mais ce sujet, remarquablement fécond, ne peut être développé ici.

Dans la pensée de Heidegger, le terme Dasein est également central parce qu'il caractérise l'être humain comme un lien entre plusieurs éléments. Loin d'être une instance finie autonome, le Dasein est un être mixte, une combinaison, juxtaposition ou concaténation de différentes entités ou notions, dont la teneur exacte semble varier selon les points de vue de Heidegger ou de ses élèves. Dans Être et Temps, la toute première définition en est encore relativement classique vis-à-vis de la philosophie occidentale : le Dasein est ce qui est nous-mêmes et qui peut s'interroger ${ }^{15}$. Quelques pages plus loin, la spécificité de la pensée de Heidegger s'affirme : le Dasein est un étant (élément de la création, dans la langue religieuse) à nul autre pareil parce qu'il entretient une relation d'être avec l'être : l'être dont Heidegger fait sa quête ne peut être dégagé que par une " analytique existentiale du Dasein $»^{16}$, c'est-à-dire que le Dasein " contient » l'être d'une manière ou d'une autre. Les définitions et caractérisations, de plus en plus élaborées, données dans Être et Temps font ressortir deux pôles : un pôle mondain (dans les deux sens du terme : à la fois banal et tenant du monde environnant) et un pôle plus secret, existentiel, bref lié à l'être même. Retracer ces définitions reviendrait à refaire l'ouvrage. Notons néanmoins que, par exemple page 53, Heidegger donne trois "points de vue " possibles au Dasein: il s'agit d'un étant (Seiende), d'un "dans le monde ", et d'un "dans être ». La facette "dans être " occupera de plus en plus Heidegger, donnant à son œuvre plus tardive son cachet de plus en plus mystique.

Si cette définition bipolaire de l'être humain brièvement esquissée évoque fortement la conception soit chrétienne, soit religieuse au sens premier du terme (religio étant ce qui attache ou relie), le tournant (Kehre) de Heidegger lui a peu à peu adjoint une 
dimension nouvelle, spécifiquement mystique : le refus de plus en plus radical de tout l'étant en tant que prérequis de la question de l'être.

Les mystiques issues de la Bible insistent sur l'importance du rejet, de l'éloignement vis-à-vis du monde jugé mauvais. L'être humain y est frappé par la déréliction, le fait d'être abandonné à la surface de la Terre, à l'image du cri de désespoir de Caïn : «Je vais errer et fuir à la surface du monde $»^{17}$. Portée à son comble, la déréliction engendre un mode de pensée ou une religion gnostique comme l'a montré Hans Jonas ${ }^{18}$ - à ceci près que le terme "gnostique " n'est usité dans l'historiographie que pour décrire certaines formes religieuses apparentées au christianisme, alors que ses mécanismes intellectuels peuvent concerner, et de fait ont concerné, d'autres religions, notamment le judaïsme.

Dans la langue de Heidegger, déréliction se dit "être-jeté", ou, en allemand, Geworfenheit, résultat de l'action du Wurf. Dans la Lettre, Heidegger fait une fois de plus résonner la langue allemande pour y trouver confirmation de sa thèse : le projet de l'homme, son objectif dans la vie, y est qualifié de Entwurf, littéralement l'esquisse, un terme qui, une fois décomposé en ent-Wurf, évoque l'action de "défaire le Wurf ». La finalité de l'être humain, chez Heidegger comme chez les gnostiques, est ainsi de rétablir la situation antérieure à la déréliction, somme toute, dans le langage de la mystique juive, de faire ou de contribuer au tiqqun ou à la tshuva (respectivement réparation et retour, de et à la situation initiale de l'univers c'est-à-dire Dieu).

\section{L'être humain, entre être et étant : la voix, la pauvreté}

La conception heideggérienne de l'être humain lui assigne, nous l'avons vu, un statut précaire, tant de par sa nature interne, duale et composite, que du fait de sa position entre les deux pôles de la totalité, à savoir l'être et l'étant créé ou issu de lui. Cette localisation ambivalente a donné naissance à de nombreuses descriptions, dans la mouvance heideggérienne et dans les religions monothéistes en général, et ce, avec un style pouvant aller du plus rationnel et abstrait jusqu'au plus poétique en passant par le plus prosaïque. Les notions auxquelles ces conceptions renvoient semblent profondément ancrées dans notre condition humaine et peuvent donc être aisément associées à des éléments de la vie quotidienne, donnant naissance à un riche champ d'images et métaphores potentielles : électricité, sphères de feu, souffle vivifiant, etc. Ces notions se retrouvent dans des courants mystiques fort éloignés, bien au-delà des frontières de telle ou telle tradition ${ }^{19}$. Par exemple, la Rose-Croix utilise dans son enseignement l'image de l'électricité pour rendre compte de sa conception de l'être humain, très proche de celle de Heidegger : nous serions telles des ampoules branchées en série, brillant du fait de la circulation de l'être, et s'éteignant une fois le filament consumé - notons qu'en hébreu l'électricité est linguistiquement liée au feu de la divinité et que, dans plusieurs traditions de la kabbale, le divin proprement dit est le fluide qui circule entre les dix sefirot. Au demeurant, le style de Heidegger lui-même, de sec et académique dans Être et Temps, est peu à peu devenu poétique, voire biblique, avec des termes tels que source, abri, maison, chemin, etc., ainsi qu'un mode de pensée par paraboles.

21 Avant de décrire les liens entre l'homme et l'étant, nous pouvons mentionner en premier lieu deux rapports ou deux modalités de contact entre l'être et l'être humain 
(la pensée en tant que fait d'une élite humaine fera l'objet de notre troisième partie) : la voix de l'être et l'être-pour-la-mort.

L'une des marques principales, bien qu'elle souffre quelques exceptions ponctuelles, de la religion juive ainsi que, plus nettement encore, des diverses formes de gnosticisme au sens large du terme, tient en la nature acoustique et non pas visuelle de la divinité. Le Dieu de la Bible se manifeste presque exclusivement par sa voix qu'elle soit directement entendue ou ne le soit que dans l'esprit ou le cœur d'un être humain privilégié, souvent désigné par le terme de prophète. Si cette thématique, du fait de sa richesse liée à sa position centrale dans le judaïsme, ne saurait être développée ici, une comparaison peut être esquissée avec la conception heideggérienne de la voix de l'être. Cette voix s'y présente sous deux formes majeures : Ruf et Stimme. Le Ruf, littéralement appel, est présent dès Être et Temps, par exemple dans les paragraphes 56 et $57:$ il est une manifestation forte et discontinue de l'être ou d'une instance intermédiaire telle que la conscience, visant à rappeler certaines vérités fondamentales. En cela, il ressemble fortement à l'appel gnostique, émanant de l'être et déclenchant un retournement complet de l'être humain qui le reçoit, mais également à certains épisodes bibliques spectaculaires. La Stimme peut être rendue par le terme de voix, probablement plutôt par son: elle est une production plus continue que le Ruf, mais bien plus faible, exigeant de ce fait un silence et une attitude réceptive de la part de l'être humain. Les descriptions que Heidegger en fait évoquent plus d'une fois le passage biblique de sur la voix silencieuse de $D^{2}{ }^{20}{ }^{20}$. D'autres termes peuvent être occasionnellement utilisés par Heidegger, au gré de son inspiration ou sujets d'étude : ainsi, le Fug, parole plus solennelle et forte, sorte de donation juridique du droit, dont l'inverse, Unfug, signifie sottise ${ }^{21}$. En tout cas, «la seule tâche qui incombe à l'homme est de se rendre attentif à la voix de l'Être. Semblable au berger qui dresse sa silhouette dans la plaine, l'homme doit maintenir son regard vers cet «horizon" d'où lui parviennent les voix inentendues $»^{22}$. La tâche de l'écoute, déclinée par Heidegger en de multiples jeux de sonorité (le verbe écouter, hören, étant proche en allemand de l'adjectif « dépendant » ou du verbe " obéir », comme, fait remarquable, dans beaucoup de langues mêmes non apparentées entre elles), a été élevée par ses disciples au rang de paradigme majeur du heideggerisme, comme le montre par exemple le titre du dernier ouvrage de François Fédier : Entendre Heidegger - et autres exercices d'écoute ${ }^{23}$.

L'être humain rencontre spontanément l'être divin à un autre titre encore: la mort. Dans les religions strictement monothéistes, un même être ontologique occupe deux rôles distincts, à savoir celui du créateur et du destructeur-rédempteur, deux actions dont la distinction peut parfois favoriser l'élaboration d'une ontologie duale, comportant une divinité créatrice, souvent mal connotée, car cause des souffrances et égarements $\mathrm{du}$ monde, et une divinité rédemptrice-destructrice; si l'exemple habituellement cité d'une telle dualité divine est le gnosticisme de Marcion, Heidegger semble avoir été tenté par elle en introduisant progressivement, en parallèle de l'être, le Gestell ou dispositif de la technique qui aurait donné naissance au monde mauvais. Heidegger ne paraît pas avoir séparé nettement être et Gestell, laissant à ce dernier un statut relativement flou d'instance issue de l'être. De ce fait, l'être se manifeste à l'être humain dans son authenticité lors de la mort, une situation que Heidegger désigne par une expression qui a connu un grand succès lors des querelles autour du passé politique présumé de Heidegger, Sein-zum-Tode, l'être-pour-la-mort. Comme dans la Bible, lors d'épisodes tels que celui de Jonas en route vers Ninive, la menace de la mort aide à se 
détacher du monde corrompu pour se concentrer sur les pensées saintes. Selon les mots de Heidegger, elle ouvre la voie à l'authenticité de l'être humain :

Le Sein-zum-Tode est devancement en un pouvoir-être de l'étant, dont la nature d'être est le devancement même [...]. Le devancement s'avère comme possibilité de l'entendement du plus propre pouvoir-être extérieur, c'est-à-dire comme possibilité de l'existence authentique ${ }^{24}$.

24 À l'inverse, le monde commun que Heidegger appelle « le On ", par opposition à l'initié connaissant les secrets du monde et de l'être divin, tend à oublier ou à occulter l'êtrepour-la-mort, incompatible avec sa superficialité :

Le mourir devient nivelé à une ad-venue, qui atteint certes l'être-là, mais n'appartient en propre à personne [...]. La parole mentionnée parle de la mort en tant que chute ad-venante [...]. Le On donne raison et élève la tentation de se dissimuler l'être propre du Sein-zumTode $^{25}$.

Observons simplement que la mort pour l'individu connaît un équivalent cosmique en la catastrophe, le retournement ou fin du monde, point typique des religions gnostiques également présent chez Heidegger - mais ceci n'est pas de notre propos.

Vis-à-vis de l'autre pôle, celui de l'étant, l'attitude prônée par Heidegger, à peine esquissée dans ses œuvres de jeunesse pour devenir de plus en plus présente par la suite, est le détachement. Le fait que la seconde naissance de l'homme, celle que recherchent les mouvements initiatiques ou mystiques, soit un retour aux origines implique une nécessaire suppression de l'étant qu'il a pu acquérir autour de lui. L'homme nouveau doit fondamentalement vivre dans la pauvreté, le refus actif de l'étant. L'arrière-plan religieux de cette pauvreté active est bien entendu très riche, depuis le récit de la Genèse où Adam et Ève ne possédaient rien, pas même des vêtements, jusqu'aux ordres monastiques d'aujourd'hui. Le vocabulaire religieux abonde de termes pour désigner la suppression de l'étant: mortification, jeûne, partage, « nuit active » (Saint Jean de la Croix), renonciation, charité, etc. Les linguistes font observer que l'hébreu est une langue d'une richesse exceptionnelle dans le champ sémantique de la mort ${ }^{26}$. Bref, la question du jugement à porter sur les richesses et sur l'étant en général semble être l'un des critères majeurs de distinction départageant les religiosités pour ainsi dire occidentales et celles, anciennes, lointaines ou minoritairesmystiques, dans lesquelles, telle est notre thèse, s'inscrit la pensée de Heidegger.

Ce dernier a souvent recours, surtout à partir de sa Kehre (tournant ou conversion), à un grand nombre de termes pour désigner la pauvreté : néant bien sûr, mais aussi Armut (pauvreté), le mot actif Verarmung (devenir-pauvre), diverses formules et jeux de mots (Er-eignung, Ab-bruch, Weg-nahme, Verweigerung, wesenhaftes Armwerdenlassen, etc., tous des mots exprimant le processus dynamique de l'advenir de la pauvreté). Comme le résume Pierre Boutang, un de ses disciples français, il faut, plus qu'un simple " détachement ${ }^{27}$, un véritable « esprit de pauvreté ».

28 S'il est impossible de retracer ici le parcours de la pauvreté chez Heidegger, quatre références peuvent être données. La leçon inaugurale à Fribourg en 1929 de Heidegger commence par un spectaculaire refus de possession de savoir ${ }^{28}$ : c'est à celui qui accomplit un acte de mortification que la vérité se révèle. Ici, c'est en renonçant à élaborer la question posée (" qu'est-ce que la métaphysique ?») que le néant se donne. Vom Wesen des Grundes (De l'essence du fondement) complète encore cette apologie de la négation, en ne la faisant plus procéder du néant, mais l'identifiant à l'être lui-même. L'être est la négation de l'étant; de ce fait, nier l'étant équivaut à atteindre l'être. Également, Heidegger définit dans les Beiträge (Contributions), probablement l'un des 
sommets de son oeuvre, la pauvreté comme clef de la richesse véritable ${ }^{29}$, rejoignant bien des auteurs religieux. Enfin, évoquons le discours de 1945 récemment paru en français sous le titre révélateur: La Pauvreté30.

L'esprit de pauvreté, lorsqu'il est étendu au savoir ou au domaine intellectuel ainsi que le fait progressivement Heidegger, peut déboucher sur un rapport spécifique à l'ensemble du savoir préalablement existant. Avant d'aborder ce rapport dans la pensée, ou plutôt dans le style intellectuel, de Heidegger, quelques remarques et mises en perspective nous semblent utiles.

La pauvreté spirituelle est une vertu recherchée par de nombreuses traditions religieuses, notamment mystiques, minoritaires ou non encore institutionnalisées. Le Tao-tö king la résume ainsi, parmi d'autres énoncés analogues : "Celui qui s'adonne à l'étude / Augmente de jour en jour. / Celui qui se consacre au Tao /Diminue de jour en jour ${ }^{31}$. " Chez Saint Jean de la Croix, il faut traverser la «nuit de la connaissance » pour pouvoir espérer atteindre l'union mystique. Dans le $\mathrm{Coran}^{32}$, les anges sont fiers de n'avoir d'autre science que la science donnée par Dieu. Dans les Évangiles, plus d'un passage célèbre sous-entend que la sagesse des sages n'est pas véritable : «Ce que tu as caché aux sages et aux savants, tu l'as révélé aux tout-petits ${ }^{33}$.» La religion juive, comme la plupart des autres grandes religions, mais peut-être encore plus nettement qu'elles, peut être subdivisée en un courant majoritaire, où le savoir au sens habituel du terme est valorisé, et un courant minoritaire prônant le contraire : ainsi, si le Talmud, plus tardif, plus rationalisant, contient des mots d'ordre très forts («nul ignorant ne saurait être pieux ${ }^{34}$ " ou "un savant est plus grand qu'un prophète »), chez les cabbalistes par exemple, il ne convient pas de rechercher les connaissances purement intellectuelles: ils mettent en relief "l'élément volontaire» de la religion, se rapprochant de la foi du petit peuple ${ }^{35}$; pour beaucoup de commentateurs, les traditions premières du désert se rapprochent bien plus des seconds que du premier (Tresmontant, Eliade, de Vaux, Onfray $\left.{ }^{36}\right)^{36}$.

31 Là encore, la pensée de Heidegger se range aux côtés des traditions minoritaires. Les écrits non philosophiques du jeune Heidegger résument déjà son point de vue. Il décrit ainsi à Hanna Arendt l'importance de l'attitude de pauvreté : « ne pas demander à quoi bon ni pourquoi, mais se contenter d'être $\|^{37} ;$ 《simplement être $»^{38}$. Bien entendu, elle est plus développée, argumentée et théorisée dans ses écrits académiques. Ainsi, Être et Temps renferme plus d'une mise en garde contre l'envie de tout savoir : "Die Neugier erschliesst alles und jedes, so jedoch, dass das In-Sein überall und nirgends ist« (« La curiosité cerne tout et n'importe quoi, de telle sorte néanmoins que l'être-dans est partout et nulle part ») ${ }^{39}$. Les sciences, procédant d'une logique accumulative, échouent à savoir l'essentiel ${ }^{40}$ : selon une sentence bien connue de Heidegger ayant suscité de nombreux débats, elles «ne pensent pas ».

Au-delà des exemples de la position générale de Heidegger, quelques précisions peuvent être portées sur ses implications philosophiques. La pensée occidentale majoritaire a entretenu essentiellement trois types de rapports avec les sagesses ou connaissances antérieures: soit une attitude polémique, dont l'enjeu est d'en abolir certaines, notamment en invoquant le critère éliminatoire vrai-faux tel que décrit par Foucault; soit une position exégétique, où la pensée nouvelle se déploie à partir de ses prédécesseurs tout en étant d'une nature différente et ne leur faisant donc pas concurrence; soit enfin une relative indépendance par la création (recherche). Heidegger et ses élèves ont pour coutume de résumer leurs rapports avec les autres 
penseurs et pensées par le terme allemand d'Auseinandersetzung, que l'on pourrait traduire par confrontation. Le terme est souvent décomposé en Aus-ein-ander-setzung, littéralement «le fait d'asseoir autre chose à partir d'une chose». L'école philosophique française de l'après-guerre, sous l'inspiration de Heidegger, utilise quant à elle le terme « déconstruction » : la pensée existante est traitée, parfois avec un grand soin et une remarquable érudition, pour montrer, « dévoiler » (Bourdieu et Heidegger) l'inanité de ses rouages. Une simple étude de la forme des très volumineux écrits de Heidegger suffit à faire état d'une caractéristique semi-constante de la pensée de Heidegger, à savoir le fait qu'elle prend son essor, non de manière autonome, mais à partir d'un auteur ou d'une citation : sa grande dépendance vis-à-vis de la philosophie ou de l'histoire de la philosophie s'accompagne par ailleurs d'un fort ou - selon le terme généralement usité - d'un radical rejet de celles-ci. Rejet qui culmine ou affirme culminer, plus que dans une opposition guerrière, en un sage détachement, que l'on pourrait qualifier par l'un des termes préférés du Heidegger de la maturité : la sérénité (Gelassenheit).

\section{La pensée, ou l'action par excellence de l'homme}

À l'issue des présents développements sur la nature et la position de l'être humain dans certaines traditions et chez Heidegger, il convient de proposer, au moins sommairement, ce que l'être humain peut de lui-même entreprendre dans la pensée heideggérienne. Le climat de l'après-guerre, marqué par l'existentialisme et la nécessité de l'engagement, a naturellement sollicité cette facette de l'enseignement de Heidegger, souvent de manière remarquablement féconde. Ainsi, la critique voire le rejet de la science ou de la pensée rationnelle ont trouvé leur homologue philosophique chez un penseur revalorisant l'art en tant que chemin vers la vérité, ne condamnant pas la marginalité, encourageant le rêve, l'extase et véhiculant enfin une conception de l'être humain non-standard, composite, susceptible de jeux ou d'effets stylistiques, bref " toujours différente à elle-même » selon une expression de l'époque. Chez Heidegger même, cette activité de séparation, de différenciation, de " différance " pour reprendre Derrida, que l'homme est incité, voire tenu de faire est dénommée pensée (Denken). Un terme aussi courant mérite plus d'une précision, notamment quant à son ressort premier et sur ses modalités.

L'activité intellectuelle, dans la tradition occidentale, prend appui sur des connaissances extérieures à son auteur, contenues notamment dans des livres. La tradition intellectuelle dans laquelle s'inscrit Heidegger rejette au contraire cette vision de l'œuvre en tant que support ou contenant de la vérité. Schopenhauer, parfois tenu pour le grand-père philosophique de Heidegger, a placé au cœur de son activité philosophique le rejet des hommes et des livres. Ainsi, l'Art de ne pas lire (au titre français éloquent, en fait le chapitre XXIV des Parerga) dénonce la lecture : celle-ci est un mauvais mode de philosophie, puisque "quand nous lisons, un autre pense pour nous » et que certains «ont lu jusqu'à s'abêtir $»^{41}$. La lecture ne laisse pas de traces positives dans l'esprit, tant que cet esprit n'est pas doué au préalable d'une potentia philosophique qui, elle, fait éclore les pensées philosophiques.

Au sein de ces traditions, la sagesse ou peut-être, si j'ose dire, «le Cherché », puisque leur quête tend vers l'être, celui que la tradition mystique du soufisme appelait "l'Aimé », ne se trouve donc pas dans un quelconque ouvrage, mais au fond de soi. 
Nous avons au début de nos développements souligné le statut spécifique de l'être humain, de l'être-là, entre l'étant et l'être: précisément son statut lui permet de poursuivre cette quête dans son intériorité même. Comme le résume Roger Munier dans sa préface à la traduction de la Lettre, « cet étant par qui l'Être a le pouvoir d'êtrelà, de se produire comme éclaircie ${ }^{42}$. L'une des difficultés de cette revendication tient en les contraintes de l'exercice universitaire de la philosophie auxquelles étaient soumis Heidegger et la plupart de ses disciples français et qui transparaissent dans la forme, très conventionnelle, des premières œuvres de Heidegger. Munier y répond en reprenant l'argument de Beaufret, au demeurant très heideggérien, c'est-à-dire opposé à la tradition issue de la Grèce classique, selon lequel la pensée ne serait pas contenue dans les écrits de Heidegger, mais simplement suggérée par ceux-ci :

Si l'analytique développée dans Sein und Zeit [Être et Temps] porte la dénomination d'existentiale, c'est précisément pour indiquer que cette description de l'être de l'homme n'a pas sa fin en elle-même, mais est ordonnée tout entière à l'expérience de l'Être dont elle ne veut que définir les approches ${ }^{43}$.

Issue d'une quête intérieure ou intériorisée, la pensée au sens heideggérien du terme se déploie selon des modalités spécifiques, dont quatre peuvent être présentées ici.

En premier lieu, si la pensée est, selon l'expression soufie, une quête du dedans, elle est, selon l'expression de Heidegger abondamment reprise par la suite, une "pensée de l'être ", la richesse polysémique en étant contenue dans les différents sens de la particule «de», qui peuvent lui conférer au moins deux significations principales. D'une part, " pensée de l'être » peut évoquer, notamment à des universitaires, au même titre que par exemple " étude de la matière " ou " exégèse d'un texte », le fait que la pensée ait pour objet fondamental l'être : le démontrer est l'objectif du début d'Être et Temps. Cependant, Heidegger n'entend pas être un spécialiste de l'être au même titre que d'autres sont spécialistes de la matière ou de tel ou tel texte : l'être ne saurait être un objet d'étude extérieur, comme le veut la tradition issue de la Grèce classique. De là, l'autre sens du « de » qui devient manifeste au heideggérien quelque peu avancé dans son apprentissage - pour ne pas dire initiation : la pensée est une émanation de l'être, au même titre que le texte sacré est issu, selon des modalités variables, de l'être divin dans les grandes religions monothéistes. Cette ambivalence du " de ", dans la présente proposition et dans d'autres encore, a été théorisée par les Heidegger studies par des développements sur les génitifs subjectifs et objectifs. Heidegger résume :

En un mot, la pensée est la pensée de l'Être. Le génitif a un double sens. La pensée est de l'Être, en tant qu'advenue par l'Être, elle appartient à l'Être. La pensée est en même temps pensée de l'Être, en tant qu'appartenant à l'être, elle est à l'écoute de l'Être ${ }^{44}$.

Notons ce qu'une telle affirmation a de proche avec le culte divin préconisé par Maïmonide, dont une traduction allemande du Guide date des débuts de la carrière de Heidegger et semble lui avoir donné plus d'une source d'inspiration ${ }^{45}$.

Deuxièmement, si la pensée est pensée de l' Être, elle détient une puissance bien supérieure à celle des simples processus mentaux, voire biochimiques liés à une activité intellectuelle habituelle. En cela, la conception heideggérienne rejoint parfaitement les attentes de ses contemporains, notamment du point de vue de l'impératif d'action spécifique à l'époque. Heidegger et les siens se sont attachés à montrer de multiples façons la nature active de la pensée, par exemple en jouant sur le mot allemand handeln, agir, proche de hand, la main, et qui signifierait prêter la main à l'être par la pensée, tout en renvoyant à divers concepts heideggeriens (Vorhandenheit, etc.). La Lettre sur l'Humanisme commence par cet appel à l'action : « Nous ne pensons pas encore de façon 
assez décisive l'essence de l'agir » et rappelle que la pensée agit de par le fait qu'elle pense (»das Denken handelt, indem es denkt «) ${ }^{46}$. Ailleurs, Heidegger décrit la pensée par des termes très concrets, plus évocateurs des actes de la vie quotidienne que de l'activité intellectuelle : entrer dans, rassembler, défaire, habiter... (notamment dans Zur Seinsfrage $e^{47}$.

Troisièmement, la pensée, loin d'être un ensemble de propositions statiques tenues pour vraies à l'instar des manuels de mathématiques, est chemin. Heidegger rejoint ici pleinement une riche thématique biblique, ou religieuse au sens général; un signe qui ne trompe pas est la reprise par ses disciples d'images pour le moins évocatrices de leur origine géographique et culturelle, telles que l'assimilation du penseur au «berger de l'être "; l'affirmation de la nécessiter d'aller et de revenir définie comme "puiser à une même source "; le fait que cheminer avec Heidegger arrache au "troupeau " du commun des mortels (Beaufret) ; l'injonction à chercher la «trace» de l'être, à écouter sa « voix silencieuse». Inutile de multiplier les références religieuses de la notion de cheminement. Selon Mircéa Eliade, les ascètes doivent être en marche permanente, toujours en mouvement ${ }^{48}$. Tresmontant et Attali montrent que l'errance permanente est le propre des Israélites. Il en est de même des mystiques juifs et chrétiens. Le prophète de Khalil Gibran se dit à plusieurs reprises toujours à la recherche du chemin le plus solitaire ${ }^{49}$. L'encyclique Deus Caritas est parle elle aussi du « chemin de montée et de purification $»^{50}$. Chez Heidegger, le cheminement est une notion centrale, jusque dans le sous-titre de ses œuvres complètes : »Wege - nicht Werke« (Chemins - non pas œuvres), ou dans le titre Holzwege, en français Chemins qui ne mènent nulle part.

41 Enfin, la pensée au sens heideggérien du terme présuppose un certain état personnel, pour ne pas dire mental, à partir duquel elle peut seulement se constituer. Les historiens de la philosophie soutiennent que cette dernière s'est constituée en Europe sur un point de vue inverse, à savoir que son exercice n'exige que l'usage de la raison ou d'autres facultés humaines universellement répandues. Des exemples souvent cités peuvent en être le système cartésien, qui reconstitue l'intégralité du monde d'une manière telle que quiconque peut l'imiter, ou l'épisode de l'esclave dans le Ménon que l'on peut lire sous l'angle de l'universalité des aptitudes à la philosophie. Cette dernière n'est pas l'apanage d'une élite sociale ou initiatique, mais résulte des facultés dont chacun est détenteur.

«Ne dépasse pas la métaphysique qui veut »: ce mot d'ordre fréquemment invoqué par Beaufret, «l'ambassadeur» de Heidegger en France, montre l'importance de facteurs propres à une personne donnée dans le succès ou l'échec de son entreprise. Heidegger lui-même, s'il semble ne pas théoriser les différences d'aptitudes entre les êtres humains, s'appuie dans l'élaboration de sa pensée sur un petit nombre d'auteurs tenus pour être plus près de l'être que les autres - une «élite » que Beaufret intégrera dans une sorte de philosophie de l'histoire dont elle sera le fil conducteur ${ }^{51}$. Heidegger fait parfois allusion aux aptitudes de chacun :

[...] eux-mêmes ont renoncé à toute possibilité de croyance dans la mesure où ils sont devenus incapables de chercher Dieu. Ils ne sont plus capables de chercher, parce qu'ils ne sont plus capables de penser ${ }^{52}$. abondamment documenté, souvent moqué, et ne nécessite donc guère plus qu'un bref rappel. Pour Heidegger, la langue grecque, de même qu'un allemand des origines reconstitué par ses soins, possède un rapport unique à l'être : penser quelque chose en 
grec revient à le penser dans sa vérité et faire résonner en soi un mot grec, à la manière des Grecs, revient à y entendre la voix de l'être. D'autres langues, telles que le français, sont bien moins privilégiées, au point d'avoir mérité cette phrase issue du zèle de Beaufret, pourtant francophone: "l'allemand a ses possibilités et le français a ses limites ». En tout cas, et ce point mériterait plus de développements pour pouvoir être dit ici, le statut du grec chez Heidegger ressemble à s'y méprendre à celui des langues sacrées dans certaines cultures, que ce soit le latin dans la chrétienté, le guèze en Éthiopie ou, surtout, l'hébreu dans la tradition juive, cette langue divine des origines, mais aussi celle des maitres de vérité dont l'usage profane a longtemps été repoussé pour en éviter les abus ainsi que, peut-être, les pouvoirs inconnus ${ }^{53}$.

\section{Conclusion}

Il est des secrets, notamment des "secrets de fabrication », que l'on a bien peine de tenir. Les ressemblances profondes entre Heidegger et plusieurs traditions religieuses anciennes, dans la plupart des thématiques abordées au sein de sa volumineuse œuvre, sont à plusieurs égards trop fortes pour passer inaperçues. Pourtant, si les études comparant Heidegger à des penseurs de l'Extrême-Orient commencent à se multiplier, notamment aux États-Unis, mettre en lumière ses analogies avec la pensée juive demeure une démarche peu fréquente, probablement en raison de la violence de la querelle autour du passé de Heidegger qui, en plus de tendre à repousser au second plan toutes les autres questions, a fait paraître inenvisageable une quelconque parenté intellectuelle de ce type. La fragmentation des disciplines universitaires, les spécialistes de la philosophie n'étant pas toujours ceux de la philologie - en particulier non européenne - et vice-versa, n'a pas favorisé les investigations. Enfin, signe troublant, plusieurs élèves français de Heidegger ont explicitement interdit toute étude de ce type: Beaufret prohibait ainsi solennellement ne serait-ce que d'envisager la Bible en hébreu - une position qui pourrait ne pas avoir pour simple cause un antisémitisme primaire, comme il est souvent dit. De même, Munier se pose cette question dans son introduction à la Lettre sur l'Humanisme et y répond à sa façon, c'est-à-dire en l'occultant :

Parvenue en ce point, une question naturellement se pose à la pensée: dans cet accomplissement de l'essence de l'homme, une échappée est-elle ouverte vers le Dieu des révélations positives et plus précisément vers celui d'Abraham, d'Isaac et de Jacob? La pensée qui tente de replacer l'homme en présence de l'Être lui-même est-elle, en son fond, religieuse? Vouloir en décider sur les seules bases que nous possédons serait prématuré et surtout peu conforme à l'intention de Heidegger. À première vue, l'idéal humain vers lequel semble s'orienter l'auteur de la Lettre et ses écrits postérieurs fait davantage penser à celui de la Grèce antique, de la Grèce sacrale d'avant le rationalisme naissant, toute pleine encore de la présence des dieux, telle que l'ont pressentie Nietzsche et surtout Hölderli ${ }^{54}$.

La pensée de Heidegger n'a donc pas de parenté juive, car Heidegger le dit.

La présente étude ne saurait être mieux qu'une esquisse, un éclairage sur un sujet bien trop vaste. Les deux figures majeures de l'être humain qui y ont été présentées, l'une étant celle de la tradition majoritaire en Occident et issue de la Grèce classique, l'autre paraissant avoir été répandue à travers le monde et s'être maintenue dans les courants mystiques et minoritaires des religions, en particulier du judaïsme, font vraisemblablement partie d'une séparation plus englobante de la philosophie en deux 
grandes modalités. Une summa divisio de la pensée humaine susceptible de s'apparenter à deux manières d'être.

\section{NOTES}

1. Genèse 1:27. Klein soutient que le mot tselem, avant de signifier "image ", aurait eu pour sens originel "something cut off", une étymologie correspondant peut-être plus encore à la suite de notre présentation. Ernest Klein, A Comprehensive Etymological Dictionary of Hebrew for Readers of English, Haifa, University of Haifa Press, 1987, p. 548.

2. Erich Auerbach, Mimésis: la représentation de la réalité dans la littérature occidentale, Paris, Gallimard, 1968.

3. Le livre fondateur d'Anthony Downs, An Economic Theory of Democracy, date de 1957. Cet ouvrage, au lieu de proposer une théorie du politique et de la sociologie politique à partir des concepts ainsi que du vocabulaire habituels à la science politique, la réinterprète en s'appuyant sur la thèse de la rationalité de l'électeur-sujet. La vie politique s'y présente donc comme agrégation de choix purement individuels, comme en microéconomie. L'«individualisme méthodologique » de tout un courant des sciences sociales était né.

4. Herbert Marcuse, Éros et Civilisation, contribution à Freud, Paris, Éditions de Minuit, 1963, pp. 12-15 et 102-103.

5. Marcel Detienne, Les Maîtres de vérité dans la Grèce antique, Paris, Maspéro, 1994, p. 131.

6. Ibid. p. 155.

7. Ron Naiweld, «Au commencement était la pratique », dans le présent numéro de Yod.

8. Pour n'en citer qu'une: Cai Werntgen, Kehren: Martin Heidegger und Gotthard Günther europäisches Denken zwischen Orient und Okzident, München, Wilhelm Fink, 2006.

9. Ibid. p. 278.

10. Mircea Eliade, La nostalgie des origines - méthodologie et histoire des religions, Paris, Gallimard, 1969, p. 12.

11. Nous voyons ici à l'œuvre un stratagème central à l'œuvre de Heidegger: la Grèce qu'il célèbre, pense et fait penser est la Grèce "première ", parfois qualifiée de "présocratique ", contrairement à la Grèce dans son acception habituelle, celle de Platon et Aristote. Ainsi, Heidegger pouvait-il s'ériger en héraut majeur d'une Grèce originelle et donc plus authentique que les autres, tout en défendant des idées et thèses plus anciennes que la Grèce, c'est-à-dire non grecques, en particulier orientales ou juives.

12. Martin Heidegger, Lettre sur l'Humanisme, Paris, Aubier, 1983, pp. 45-47.

13. Terme qualifiant l'œuvre de certains auteurs qui accordaient une place jugée excessive au sujet individuel dans les sciences de l'esprit.

14. Par exemple, la traduction allemande du Guide des Égarés de Adolf Neiss (1923 et 1924) décrit la pensée de Maïmonide et de Duns Scotus comme »die Lehre vom Dasein und der Einheit Gottes«, « l'enseignement du Dasein [c'est-à-dire de l'existence effective] et de l'unité de Dieu » (Mose Ben Maimon, Führer der Unschlüssigen, Leipzig, Felix Meiner, tome I, p. CLI).

15. Martin Heidegger, Sein und Zeit, Tübingen, Max Niemeyer, 2001, p. 7.

16. Ibid. p. 13.

17. Genèse 4:14.

18. Hans Jonas, La religion gnostique, Paris, Flammarion, 1978. 
19. Voir par exemple Rudolf Otto, Mystique d'Orient et mystique d'Occident, Paris, Payot, 1996.

20. «Et il se fit un vent grand et violent, déchirant les montagnes et brisant les rochers devant l'Éternel : l'Éternel n'était pas dans le vent. Et après le vent, un tremblement de terre : l'Éternel n'était pas dans le tremblement de terre. Et après le tremblement de terre, du feu: l'Éternel n'était pas dans le feu. Et après le feu, un son à peine perceptible. Et quand Élie l'entendit, il cacha sa face dans son manteau et sortit et se tint à l'entrée de la caverne. Et voici, une voix lui parla. » (I Rois 19:11-13).

21. Un autre exemple de jeu de mots typiquement heideggérien porte sur la Stimme. Comme on sait, l'être-là est défini par Heidegger en tant que souci (Sorge), non pas un souci apparenté à l'angoisse que connaît la psychopathologie, mais le souci de l'être («tout souci est souci de l'Etre ", dit la Lettre p.13), dans une signification qu'on peut rapprocher de la «frayeur » éprouvée par les haredim de la religion juive, ou plus globalement de l'effroi mystique dont Rudolf Otto faisait un des trois éléments du sacré. Ce souci doit être généralisé pour mener l'êtrelà à l'authenticité, il doit faire ambiance, c'est-à-dire Stimmung en allemand, un mot qui fait résonner le terme Stimme, voix.

22. Lettre sur l'Humanisme, op. cit., pp. 13-14.

23. Paris, Le Grand Souffle, 2008.

24. Être et Temps pp. 262-263.

25. Ibid. p. 253.

26. Par exemple : Yishaï Neuman, Hébreu, Presse israélienne - pratique de la langue, cours et devoirs, Poitiers, CNED, pp. 139 et 140.

27. Pierre Boutang, Ontologie du secret, Paris, PUF, 1973, p. 322.

28. »Was ist Metaphysik? « - Die Frage weckt die Erwartung, es werde über die Metaphysik geredet. Wir verzichten darauf. Statt dessen ... («Qu'est-ce-que la métaphysique?» La question suscite l'attente du fait qu'on traitera de la métaphysique. Nous y renonçons. À la place...).

29. »[...] nicht das Bedürfen, nicht Mittellosigkeit [...] nicht Entbehren, nicht Angewiesenheit auf das Entzogene, aber auch nicht in blosser Umkehrung ein Reichtum, sondern die Er-eignung des eigenen Wesens des Seyns« (« [...] non le besoin, non l'absence de ressources [...] non le fait de manquer, non la dépendance vis-à-vis du retiré, mais pas non plus dans un simple retournement une richesse, mais l'ad-venue de l'être même de l'Être "), avec une graphie supposément archaïque de Sein pour éviter d'en écrire le Nom, à l'image de la substitution de Eloqim à Elohim par les juifs pieux (Beiträge VIII § 100).

30. Philippe Lacoue-Labarthe, La pauvreté (Die Armut), Strasbourg, Presses universitaires de Strasbourg, 2004.

31. Lao Tseu, Tao-tö king, Paris, Gallimard, 1990, chap. XLVIII.

32. 2, 30 .

33. Matthieu 11:25.

34. Avot 2, 6.

35. Gershom Scholem, Les grands courants de la mystique juive, Paris, Payot, 1994, p. 251.

36. Voir par exemple Claude Tresmontant, Essai sur la pensée hébraïque, Paris, Cerf, 1953 ; Michel Onfray, Traité d'athéologie : Physique de la métaphysique, Paris, Grasset, 2005.

37. Lettres p. 33.

38. Ibid. p. 15.

39. Être et Temps $\S 38$ p. 177

40. Par exemple : »Ihr [des sciences] Fortschritt vollzieht sich nicht so sehr in der Aufsammlung der Resultate und Bergung derselben in " Handbüchern », als in dem aus solcher anwachsenden Kenntnis der Sachen meist reaktiv hervorgetriebenen Fragen nach den Grundverfassungen des jeweiligen Gebietes.« ( «Le progrès des sciences se déroule non pas tant dans l'accumulation des résultats et leur ensevelissement dans des «manuels », mais de par les questions portant sur les 
constitutions fondamentales de chaque domaine émanant du savoir croissant des choses. ») (Être et Temps p. 9).

41. Arthur Schopenhauer, L'Art de ne pas lire, Biarritz, Distance, 1992, pp. 9-10.

42. Lettre sur l'Humanisme, op. cit., p. 13.

43. Ibid. p. 11.

44. Ibid. p. 34-35.

45. À grands traits, nous pouvons dire que, en plus du vocabulaire propre à cette traduction (Dasein, Sein, Seiendes, Denken, Wesen, Wohnen, etc., autant de termes-clefs chez les deux auteurs), Heidegger partage avec elle plusieurs idées fondamentales. Ainsi, dans la préface, l'influence de Maïmonide sur Duns Scot (au demeurant sujet de la thèse de Heidegger) est résumée comme la transmission d'un savoir sur le Dasein et l'unité de Dieu, ainsi que l'exclusivité de l'usage du Tétragramme (p. CLI). Maïmonide aurait transmis à Nicolas de Cues le caractère outre-mondain de Dieu et la nécessité de l'approcher par ses attributs (p. CLII). Les ressemblances entre la pensée de Maïmonide et celle de Heidegger mériteraient, quant à elles, une étude spécifique.

46. Lettre sur l'Humanisme, op. cit., pp. 26-28.

47. Martin Heidegger, Zur Seinsfrage, Frankfurt am Main, Klostermann, 1977.

48. Mircea Eliade, Le Sacré et le Profane, Paris, Gallimard, 1987, p. 156.

49. Khalil Gibran, Le Prophète, Paris, Casterman, 1956, p. 82.

50. Benoît XVI, Deus Caritas est, Paris, Pierre Téqui, 2006, §6.

51. Une objection possible tiendrait en ce qu'il s'agit là d'un mécanisme communément répandu qui amène chaque universitaire à avoir un petit nombre d'auteurs préférés. Néanmoins, faiblesses humaines mises de côté, la tradition occidentale exige qu'un tel choix porte non sur les penseurs en tant que tels, mais sur certaines de leurs assertions tenues pour vraies et réutilisées ailleurs. Chez Heidegger au contraire, du fait du statut privilégié vis-à-vis de l'être d'un penseur, virtuellement toutes ses paroles seront celles de l'être et se prêteront à sa recherche, voire à une méditation respectueuse.

52. Martin Heidegger, Chemins qui ne mènent nulle part, Paris, Gallimard, 2002, p. 322.

53. Comme le dit, tout près de nous, Lévinas: "L'acte, la parole, la pensée du juif ont le redoutable privilège de détruire ou de restaurer des mondes ». Emmanuel Lévinas, Difficile liberté, Paris, Albin Michel, 2006.

54. Lettre sur l'Humanisme, op. cit., p. 20.

\section{RÉSUMÉS}

In the sixties and seventies, theoretical discussions in European social sciences focused on the nature of the human being. Proponents of the rational subject, characterizing. Enlightenmentprone Western classical philosophy, went against the advocates of the much older and lessknown anti-subjective tradition. Our study aims at giving an archaeological insight into this second paradigm, going back first to Heidegger, the master thinker of contemporary European social sciences, then to the religious - mainly Jewish - roots of his thought. It views human being not as an achieved and autonomous entity, but as an emanation of the Being. Men have to follow the path it sets and its voice, free themselves from material assets which are mere impediments, and eventually enter into a superior dimension of thinking. Beyond our limited case-study, the 
whole question of the persistence of another main - albeit forgotten - way of thinking in the very heart of modernity is being raised anew.

INDEX

Mots-clés : nature humaine, Dasein, Heidegger Martin (1889-1976), ontologie, philosophie hébraïque, être humain

Keywords : human nature, Dasein, Heidegger Martin (1889-1976), ontology, Hebrew philosophy, human being

Thèmes : philosophie

\section{מילות מפתח}

דת בשר ודם, קיום, עברית ופילוסופיה, אונטולוגיה, מרטין היידגר, להיות אדם: 